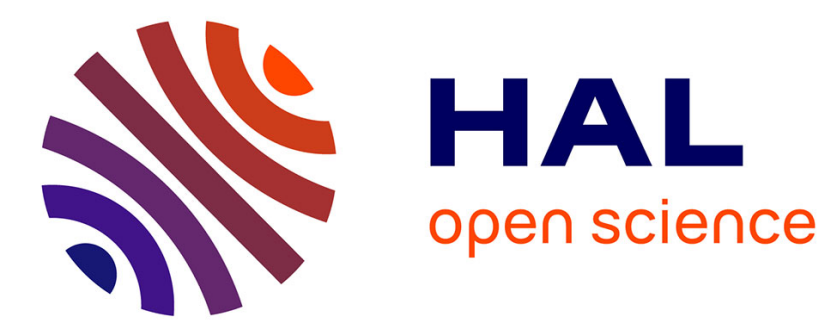

\title{
Current Practice in Management of Muscle Haematomas in Patients with Severe Haemophilia
}

Rikke Beyer, Jørgen Ingerslev, Benny Sørensen

\section{To cite this version:}

Rikke Beyer, Jørgen Ingerslev, Benny Sørensen. Current Practice in Management of Muscle Haematomas in Patients with Severe Haemophilia. Haemophilia, 2010, 16 (6), pp.926. 10.1111/j.13652516.2010.02275.x . hal-00552624

\section{HAL Id: hal-00552624 https://hal.science/hal-00552624}

Submitted on 6 Jan 2011

HAL is a multi-disciplinary open access archive for the deposit and dissemination of scientific research documents, whether they are published or not. The documents may come from teaching and research institutions in France or abroad, or from public or private research centers.
L'archive ouverte pluridisciplinaire HAL, est destinée au dépôt et à la diffusion de documents scientifiques de niveau recherche, publiés ou non, émanant des établissements d'enseignement et de recherche français ou étrangers, des laboratoires publics ou privés. 


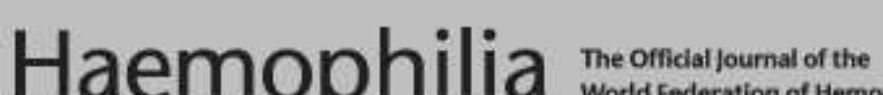 \\ World Federation of Hemophilia}

\section{Current Practice in Management of Muscle Haematomas in Patients with Severe Haemophilia}

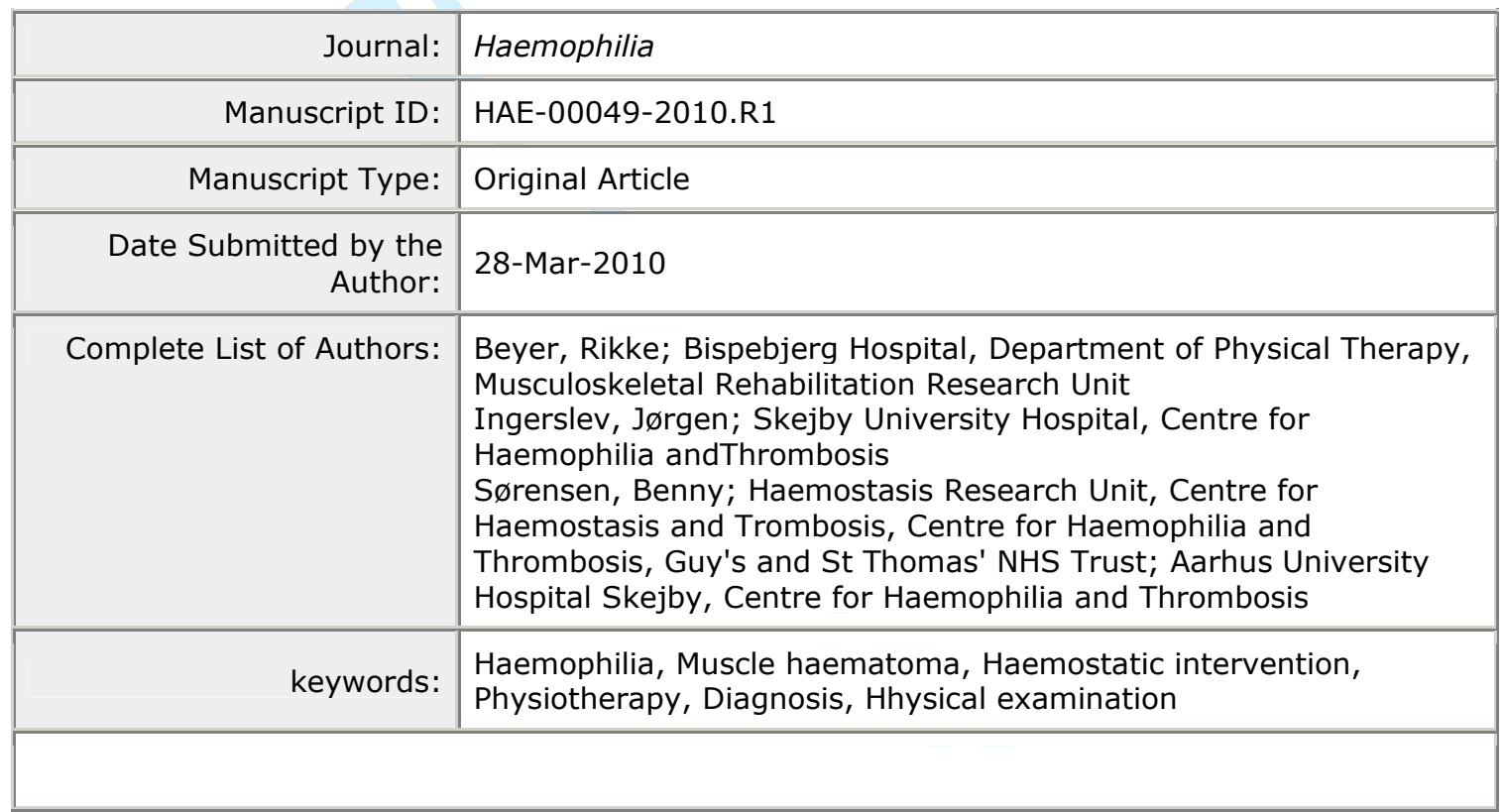

\section{S) ScholaroNE




\title{
Current Practice in Management of Muscle Haematomas in Patients with Severe Haemophilia
}

\author{
Rikke Beyer ${ }^{1,3}$, Jørgen Ingerslev², Benny Sørensen ${ }^{1,2}$ \\ ${ }^{1}$ Haemostasis Research Unit, Centre for Haemophilia and Thrombosis, Department of \\ Haematology and Oncology, Guy's and St Thomas Hospital \& NHS Foundation Trust \& King's \\ College London School of Medicine, London, UK \\ ${ }^{2}$ Center for Haemophilia and Thrombosis, Department of Clinical Biochemistry, Aarhus \\ University Hospital, Skejby, Denmark \\ ${ }^{3}$ Department of Physical Therapy, Musculoskeletal Rehabilitation Research Unit, Bispebjerg \\ Hospital, Copenhagen, Denmark
}

Key words : : Muscle bleeding, muscle haematoma, diagnosis, physical examination, haemostatic intervention, physiotherapy

Running title : Management of Muscle haematoma in Haemophilia

\section{Word count : Abstract: 250, Main text: 2123 (excluding refereces)}

\section{Disclosure}

Novo Nordisk kindly supported the study by providing an unrestricted educational grant supporting the questionnaire e-survey.

\author{
Contact \\ Benny Sørensen, MD, Ph.D, Assoc. professor \\ Senior Clinical Research Fellow \& Honorary Lecturer \\ Haemostasis Research Unit \\ Center for Haemophilia and Thrombosis, Guy's and St Thomas Hospital \& NHS Foundation Trust \\ Email: benny.sorensen@kcl.ac.uk
}




\section{Abstract}

Muscle haematomas ( $\mathrm{MH}$ ) represent $10-25 \%$ of all bleeds in patients with severe Haemophilia. We performed a cross sectional survey on current practice in management of $\mathrm{MH}$ with participation from 22 consultants. The respondents reported $492 \mathrm{MH} /$ year, corresponding an average of 25/centre, most associated with trauma. Iliopsoas (55\%), calf (18\%), and thigh (18\%) bleeds were scored as most serious. Half of respondents distinguished between contusion and strains, whereas the majority (68.2\%) did not categorise bleedings as intra- or intermuscular, although $77.3 \%$ routinely used ultrasound. Half of respondents used a standard protocol for management of MH. A total of 20 of 22 (90.9\%) offered physiotherapy in the hospital following $\mathrm{MH}$, with no clear consensus on timing and type of treatment. In a theoretical case, $70 \mathrm{~kg}$ patient with a soleus triceps haematoma, the average initial dose was $2730 \mathrm{U}$ (range: 1750-4000) twice daily for 3-5 days. In a similar case of a patient with inhibitors, $31.8 \%$ reported first line and only use of either rFVIIa or aPCC, while 36.4\% switched between bypassing agents. Using rFVIIa the median dose was $100 \mu \mathrm{g} / \mathrm{kg}$ (range: 85 - 270) and with aPCC the median dose was $70 \mathrm{U} / \mathrm{kg}$ (range: 50 - 100). The majority (68.2\%) did not use antifibrinolytics. Resolution of pain $(81.8 \%$ \& $77.3 \%$ ) was regarded the key clinical marker of arrest of bleeding as compared to diminished swelling and improved range of motion. The survey outlines limited consensus in management of $\mathrm{MH}$ in patients with Haemophilia and highlights potential topics for future studies. 


\section{Introduction}

Muscle bleedings in patients with Haemophilia often cause considerable pain and compromise overall physical capacity. Intramuscular haemorrhages are reported to represent approximately $10 \%-25 \%$ of all bleedings in patients with haemophilia [1]. In patients with severe Haemophilia, bleeds may become limb threatening. Recovery and rehabilitation may be protracted and insufficient. In contrast to spontaneous joint haemarthrosis, muscle bleeds are mostly associated with trauma [1]. In general, muscle haematomas in patients with haemophilia are often considered as difficult-to-treat bleeds. Insufficiently treated muscle bleeds may result in several complications such as e.g. irreversible damage to the muscles, including compartment syndrome, reduced range of motion, loss of function, myositis [2], damage to tendons (Volkman's ischemic contractures) [3], as well as excessive blood loss, infection, peripheral nerve complications, and development of haemophilia pseudo tumor [4]. Unfortunately, the majority of research and development within Haemophilia are focused on mechanisms related to preventing haemarthrosis and maintaining a good functional joint status. Only very few publications have reported on aspects of muscle haematoma in patients with severe Haemophilia [1;5]. Predominantly, reports have focused on management of $m$. psoas major bleeds [6;7] and haemophilia pseudo tumors [8-10]. As of today, limited consensus exists regarding choice of factor concentrate, dosages of factor substitution utilised, frequency of dosing, and duration of the haemostatic treatment. In particular, in patients with severe Haemophilia and inhibitors, studies supporting various approaches to treatment regimens do practically not exist. Finally, important information on concomitant and adjuvant treatment such as physiotherapy are rarely specified or described in detail.

The aim of the present study was to conduct a cross-sectional study on current practice in management of muscle bleeds in patients with severe Haemophilia A amongst haemophilia comprehensive care centres from UK, Denmark, Ireland, and Australia based on an electronic mail questionnaire. 


\section{Materials and Methods}

\section{Definition and classification of a muscle haematoma}

In the present study, a muscle haematoma was defined as a bleeding in any anatomical skeletal muscle group. Clinical classification and differentiation between different types of muscle haematoma are described in a recent review (ref).

\section{Questionnaire on current practice of muscle haematoma}

An electronic questionnaire was developed addressing evolvement of muscle haematoma, diagnosis of muscle haematomas, haemostatic intervention of muscle bleeds in patients with severe haemophilia without and with inhibitors, use of concomitant or adjuvant haemostatic intervention, diagnostic procedures, and physiotherapy (See Appendix 1). The questionnaire was emailed to consultant haematologists from 50 registered Haemophilia comprehensive care centres (CCC) in the United Kingdom (UK), Ireland (IR), Denmark (DK), and Australia (AUS). We advised that the questionnaire was answered by the consultant haematologist and not by junior doctors or nurses. Centres were asked to answer questions regarding adults only. Respondents reported estimated figures rather than actual measured data. Each participant was offered $£ 100$ for the effort and time spent filling out the questionnaire. All e-survey was circulated by June 2008. We allowed a period of 1 month for answering the questionnaire. Gentle reminders were emailed once a week. Following closure of the study period, results from the questionnaire were organized, analyzed and interpreted by the authors. In order to ensure the validity and quality of data, two of the investigators (RB and BS) independently listed the results in a preformed excel spreadsheet, using a predetermined checklist. In case of doubt or disagreement of the meaning of the responses, the third investigator (JI) evaluated the questionnaire. 


\section{Data analysis and statistical considerations}

Quantitative data and categorical data are presented using descriptive statistics. Qualitative statements are organized in tables, whereas quantitative measures are listed as figures and graphically illustrated.

\section{Ethical considerations}

The data from each participant are anonymous; hence, none of the results can be referred back to a specific centre. The questionnaire did not include specific patient data.

\section{Results}

\section{Frequency and aetiology of muscle bleeding}

In total 22 consultants representing 20 comprehensive haemophilia care centres in UK (17), IR (1), DK (2), and AUS (2) responded to the questionnaire. The respondents reported an estimated number of 492 muscle haematomas/year, corresponding an average of 25/CHCC. Using a score system ranging from 0-10 (0 representing never, 10 representing always) the aetiology of muscle haematomas was predominantly assessed as associated with trauma (score 7.1 of 10) rather than spontaneous development (4.0 of 10). Amongst a list of different anatomic localisations of muscle haematomas, Iliopsoas (55\%), calf (18\%), and thigh (18\%) bleeds were scored as the most serious (Figure 1).

\section{Diagnostic procedures}

Half of the respondents stated they distinguish between contusion and strains, whereas the majority $(68.2 \%)$ did not categorise bleedings as intra- or intermuscular, although $77.3 \%$ routinely used diagnostic ultrasound.

\section{Haemostatic intervention}

Only $50 \%$ of respondents reported using a standard protocol for management of muscle haematomas. 
The questionnaire outlined a theoretical case: "An objectively verified muscle haematoma in the soleus triceps of a lower limb of a 29 year old patient, weighing 70 kilos with severe Haemophilia A." followed by questions addressing i) initial dosages of factor, ii) subsequent dosages of factor, iii) duration of therapy, iv) tapering of dosages or dose frequency, as well as v) addition of an anti-fibrinolytic.

\section{Haemostatic intervention in patients with severe Haemophilia A without inhibitor}

The average initial dose of factor VIII was 2730 U (range: 1750-4000). Treatment was continued for a mean of 3-5 days (range: 1-10). Subsequent dosages were similar to the initial dosage, although the majority of respondents indicated they were tapering the dosage and dose frequency based on daily assessment of each individual case. The majority (77.3\%) of consultants did not distinguish recombinant factor VIII from plasma derived factor VIII concentrate containing von Willebrand factor. The total amount of factor VIII used ranged from minimum $=4500 \mathrm{U}$ to maximum $=50000 \mathrm{U}$ (median=10750 $\mathrm{U}$, mean=17103 U) depending on the severity, type and anatomical localisation of the haematoma.

\section{Haemostatic intervention in patients with severe Haemophilia A with inhibitors}

In a similar case of a patient with inhibitors, 31.8\% reported first line and only use of either recombinant factor VIIa (rFVIIa) or plasma derived activated prothrombin complex concentrate (pd-aPCC), while 36.4\% switched between bypassing agents. Using rFVIIa the median starting dose was $100 \mu \mathrm{g} / \mathrm{kg}$ (range: 85 - 270) and with aPCC the median dose was $70 \mathrm{U} / \mathrm{kg}$ (range: 50 - 100). Subsequent dosages ranged from 85-270 $\mu \mathrm{g} / \mathrm{kg}$ for rFVIIa (mean=110) and 50$75 \mathrm{U} / \mathrm{kg}$ (mean=63) for pd-aPCC. Dosing interval was 2-3 hrs for rFVIIa and 12$24 \mathrm{hrs}$ for pd-aPCC. The average duration of treatment was $2.3-4.4$ days, longer for pd-aPCC (4.1 days) than rFVIIa (2.8 days). 
For both patients with and without inhibitors, physicians generally expected haemostasis to be achievable within 6 hours, although up to $18 \%$ of centres allowed $>12$ hrs to obtain haemostasis (Figure 2).

\section{Adjuvant haemostatic intervention}

The majority (68.2\%) did not use concomitant antifibrinolytic treatment. The consultant reporting to use tranexamic acid, dosage regimens varied from $1 \mathrm{~g}$ daily to $25 \mathrm{mg} / \mathrm{kg}$ qds 6 hourly. The antifibrinolytic treatment was most often continued for 5-7 days.

\section{Clinical marker of arrest of bleeding and maintaining haemostasis}

Resolution of pain (patients without inhibitors: 18 out of 22 haemophilia treaters $=81.8 \%$ \& patients with inhibitors: 17 out of 22 haemophilia treaters $=77.3 \%$ ) was regarded the key clinical marker of arrest of bleeding and maintenance of haemostasis as compared to diminished swelling and improved range of motion. Figure 3, Panels A and B illustrates the scoring of importance (1 important to 4 less important) amongst patients without and with Haemophilia, showing that pain was scored as the most important marker.

\section{Physiotherapy}

A total of 20 of 22 (90.9\%) offered physiotherapy in the hospital following muscle haematomas. As outlined in Table 1, there was limited consensus on timing and type of treatment. RICE-treatment (rest-ice-compression-elevation) was recommended in most centres. All centres offered therapeutic ultrasound in the hospital setting, although there was limited agreement on the optimal timing of the intervention.

Grading of importance of further research and studies in management of muscle haematoma in patients with Haemophilia

The overall importance of further research and studies in management of muscle haematomas scored 7 and 8 (out of 10) for haemophilia patients without and with inhibitors. 


\section{Discussion}

Muscle haematomas represent difficult to treat bleeds in patients with severe Haemophilia. Multiple anatomical targets and various trauma mechanisms complicate the clinical evaluation, diagnosis, treatment and rehabilitation. Currently, limited consensus exists on the comprehensive management of muscle haematomas in Haemophilia patients.

The present survey verified that the majority of muscle haematomas has a traumatic aetiology. It may be speculated whether patients with inhibitors are more prone to develop muscle haematomas than patients without inhibitors. Some studies suggest that muscle haematomas represent $10 \%$ of all bleeds in patients with severe Haemophilia [1]. However, the data from the US home treatment study of rFVIIa suggest that up to $23 \%$ of bleeds in patients with inhibitors are muscle haematoma [11]. These questions were not explored in further detail in the present study, however, should be kept in mind for future investigations.

Not surprisingly, iliopsoas muscle bleeds were reported as the most severe type of muscle haematoma. Likewise, the majority of previous reports and studies have focused on the iliopsoas bleeds [6;7].

The study revealed sparse consistency regarding diagnostic procedures. Only half of the respondents distinguished contusions from strains. The majority of centres listed the use of ultrasound for diagnosis. However, only few differentiated between intra- vs. inter-muscular localisation of the haematoma. Diagnostic classification of muscle haematomas is a complicated clinical discipline and in the current issue of Haemophilia we have reviewed classification and strategies for optimised diagnosis of muscle haematomas [12].

Moreover, the questionnaire highlighted limited agreement regarding dosing regimens and duration of the pharmacological therapy. Only half of respondents stated to have a specific written protocol on recommendations for coagulation factor treatment of muscle bleeds. Thus, the total amount of factor VIII used 
varied considerably. The lack of consensus was even more pronounced with Haemophilia patients with inhibitors. A potential explanation for the variation in treatment regimens could be the markers of arrest of bleeding and achieved haemostasis. The respondents of the survey predominantly used pain rather than reduced swelling and improved range of motion as the marker for arrest of bleeding as well as achieved haemostasis. It is noteworthy that, even in professional athletes the entire repair process and rehabilitation period following muscle injuries may take more than 30 days [13]. Pain may be considered a physiological response to muscle damage and present despite arrest of bleeding and normal haemostasis. Hence, it seems that optimised measures for assessing ongoing bleeding and achieved haemostasis are essential for optimising treatment regimens.

The majority of participants indicated a time period of less than $6 \mathrm{hrs}$ as the preferred / accepted time to arrest of bleeding. There was a slight tendency to accept of longer time to arrest of bleeding in patients with inhibitors. This may be due to less predictability regarding haemostatic response as well as difficulties in monitoring and dosing the bypassing agents.

Recently investigations have shown that patients with severe Haemophilia not only suffer from compromised thrombin generation, but also insufficient clot stability caused by delayed and incomplete activation of FXIII [14] and increased susceptibility toward accelerated fibrinolysis [15]. Clinical studies have suggested a potent effect of concomitant antifibrinolytic treatment with tranexamic acid [15]. However, the majority of responders in the survey stated not to use adjuvant tranexamic acid for muscle haematoma. The study did not explore reasons for limited use of tranexamic acid. Lack of clinical studies may be one reason, whereas theoretical speculations on thrombotic risk may be another explanation. Further mechanistic as well as clinical studies would be desirable.

The timing and type of adjuvant physiotherapy recommended revealed considerable discrepancy. The RICE principle and therapeutic ultrasound was 
commonly recommended. However, a series of other interventions were mentioned and recommended. Obviously, it would be interesting to develop a series of systematic studies challenging various physiotherapy regimens. The current issue of Haemophilia lists some experiences from Sports Physiotherapy that may prove useful for improving overall rehabilitation and prevention of muscle injuries in patients with severe Haemophilia [12]. Recent publications have proposed a beneficial and potentially protective effect of regular exercise in patients with severe Haemophilia [16]. Optimized muscular function may protect against joint bleedings and improved fitness was associated with a higher rating of quality of life [17-19]. Likewise, a recent study describe a conservative and non-operative physiotherapeutic regime for the management of chronic haematomata and pseudotumors in patients suffering from haemophilia [20].

The study contains a series of important limitations. This cross sectional study only provides a random sample of current management of muscle haematomas. The nature of the study design does not provide valid data on causality, neither on actual measured frequencies and dose regimens. Respondents may have reported on their preferred/wished treatment modality, with no assurance that the data reflect actual practice. As an example, the numerous types of physiotherapy may reflect what consultant haematologists would like to offer, rather than what is actually available. The questionnaire was sent to 50 random comprehensive haemophilia care centres, however, only centres from UK, Ireland, DK, and Australia were enrolled. Thus, selection and information bias may be present and the data has reduced external validity.

In conclusion, the present survey suggests the need for further studies on the pathology, diagnosis, and importance of early treatment to achieve rapid control of bleeding, as well as rehabilitation following muscle haematomas in patients with Haemophilia. A prospective international multicentre registry of current management of muscle haematomas seems desirable to get more data. Optimised and systematic diagnosis may facilitate a better strategy regarding type and timing of physiotherapy. 


\section{Acknowledgement}

We are grateful to colleagues at comprehensive haemophilia care centres in UK, DK, Ireland, and Australia for their time and effort in responding to our questionnaire.

\section{Appendix}

A pdf-copy of the questionnaire can be obtained by emailing the corresponding author.

\section{References}

\section{Reference List}

1. Alcalay M, Deplas A. Rheumatological management of patients with hemophilia. Part II: Muscle hematomas and pseudotumors. Joint Bone Spine 2002; 69(6): 556-9.

2. Brukner P, Khan K. Clinical sports medicine. 3rd ed. Australia ed. McGrawHill; 2006.

3. Lancourt JE, Gilbert MS, Posner MA. Management of bleeding and associated complications of hemophilia in the hand and forearm. $J$ Bone Joint Surg Am 1977; 59(4): 451-60.

4. Ahlberg AK. On the natural history of hemophilic pseudotumor. J Bone Joint Surg Am 1975; 57(8): 1133-6.

5. Aronstam A, Browne RS, Wassef M, Hamad Z. The clinical features of early bleeding into the muscles of the lower limb in severe haemophiliacs. $J$ Bone Joint Surg Br 1983; 65(1): 19-23.

6. Dauty M, Sigaud M, Trossaert M, Fressinaud E, Letenneur J, Dubois C. Iliopsoas hematoma in patients with hemophilia: a single-center study. Joint Bone Spine 2007; 74(2): 179-83.

7. Heim M, Horoszowski H, Seligsohn U, Martinowitz U, Strauss S. Ilio-psoas hematoma--its detection, and treatment with special reference to hemophilia. Arch Orthop Trauma Surg 1982; 99(3): 195-7.

8. NELSON MG, MITCHELL ES. Pseudo-tumour of bone in haemophilia. Acta Haematol 1962; 28: 137-44. 
9. Grauthoff $\mathrm{H}$, Hofmann $\mathrm{P}$, Lackner $\mathrm{K}$, Brackmann HH. [Haemophilic pseudo-tumours and iliac haematomas: radiological and clinical findings (author's transl)]. Rofo 1978; 129(5): 614-20.

10. Chakal F, Viso R, Fernandez PF. Percutaneous treatment of haemophilic digital pseudo tumours. Int Orthop 2005; 29(3): 197-8.

11. Key NS, Aledort LM, Beardsley D, Cooper HA, Davignon G, Ewenstein BM, et al. Home treatment of mild to moderate bleeding episodes using recombinant factor VIIa (Novoseven) in haemophiliacs with inhibitors. Thromb Haemost 1998; 80(6): 912-8.

12. Beyer R, Ingerslev J, Sørensen B. Muscle Bleeds in Professional Athletes Diagnosis, Classification, Treatment, and Potential Impact for Patients with Haemophilia. Haemophilia 2010; (Manuscript in submission).

13. Mason DL, Dickens V, Vail A. Rehabilitation for hamstring injuries. Cochrane Database Syst Rev 2007; (1): CD004575.

14. Brummel-Ziedins KE, Branda RF, Butenas S, Mann KG. Discordant fibrin formation in hemophilia.J Thromb Haemost 2009; 7(5): 825-32.

15. Hvas AM, Sorensen HT, Norengaard L, Christiansen K, Ingerslev J, Sørensen B. Tranexamic acid combined with recombinant factor VIII increases clot resistance to accelerated fibrinolysis in severe hemophilia A. J Thromb Haemost 2007; 5(12): 2408-14.

16. Buzzard BM. Physiotherapy, rehabilitation and sports in countries with limited replacement coagulation factor supply. Haemophilia 2007; 13 Suppl 2: 44-6.

17. Wittmeier K, Mulder K. Enhancing lifestyle for individuals with haemophilia through physical activity and exercise: the role of physiotherapy. Haemophilia 2007; 13 Suppl 2: 31-7.

18. VON MS. Quality of life and sports activities in patients with haemophilia. Haemophilia 2007; 13 Suppl 2: 38-43.

19. van der Net J, Vos RC, Engelbert RH, van den Berg MH, Helders PJ, Takken T. Physical fitness, functional ability and quality of life in children with severe haemophilia: a pilot study. Haemophilia 2006; 12(5): 494-9.

20. D'Young AI. Conservative physiotherapeutic management of chronic haematomata and haemophilic pseudotumours: case study and comparison to historical management. Haemophilia 2009; 15(1): 253-60. 


\section{Figures}

Figure 1

Anatomical localisation of most severe muscle haematoma

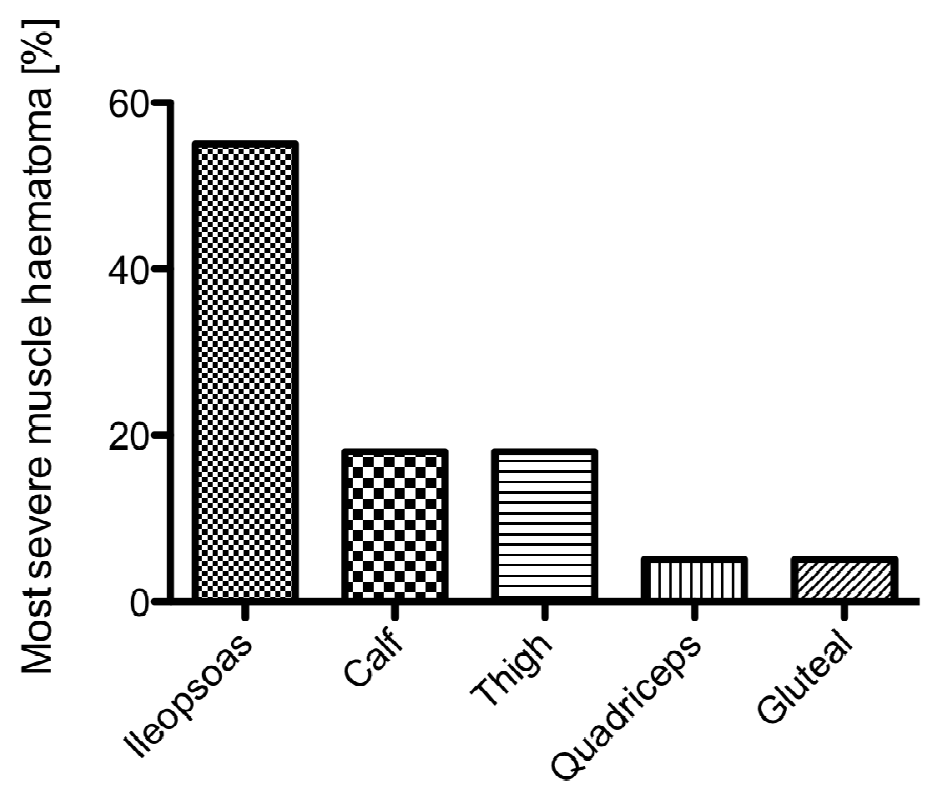


Figure 2

Accepted time to arrest of bleeding

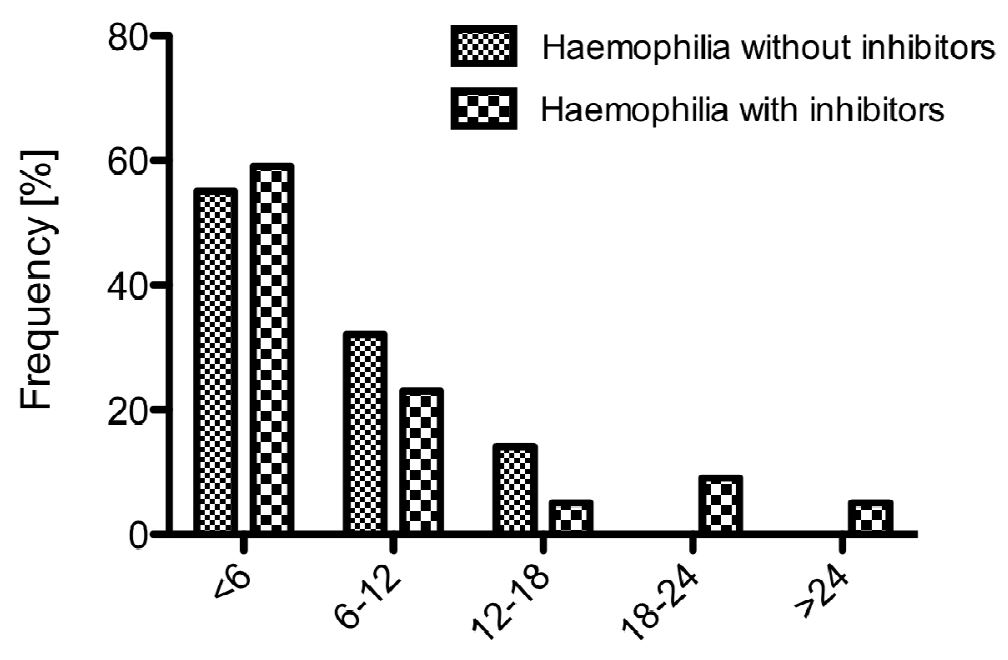


Figure 3

Markers of arrest of bleeding and maintaining haemostasis

A

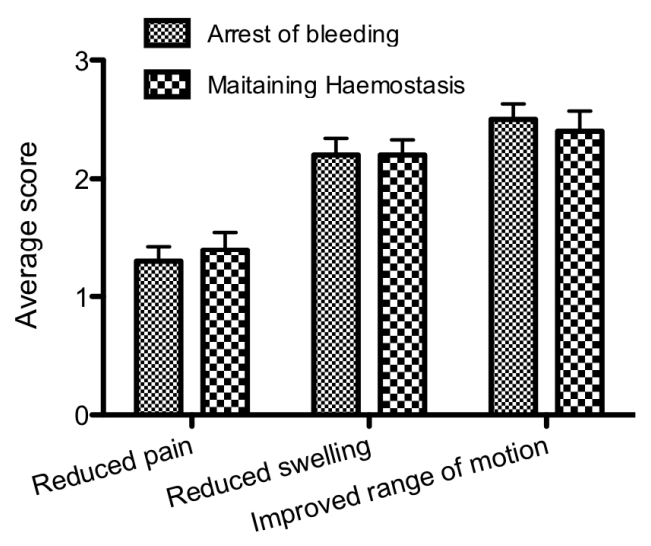

B

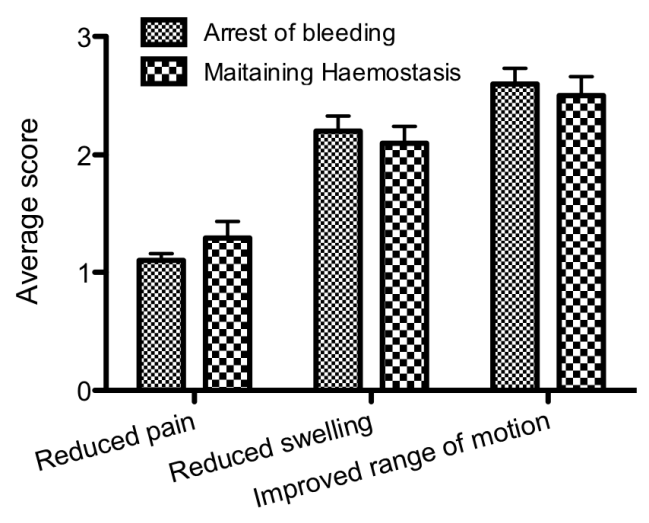

Panel A: Haemophilia patients without inhibitors

Panel B: Haemophilia patients with inhibitors 


\section{Legends}

\section{Figure 1}

Anatomical localisation of most severe muscle haematoma

Illustrates frequency data (\%) on the anatomical localisation of the most severe muscle haematoma.

\section{Figure 2}

Accepted time to arrest of bleeding

Depicts the accepted time to arrest of bleeding in patients without and with inhibitors.

\section{Figure 3}

\section{Markers of arrest of bleeding and maintaining haemostasis}

Illustrates the results of score of importance of the three clinical markers: reduced pain, reduced swelling, improved range of motion as defined by subjective reported relief of pain, reduced circumferential measure, and measurement of the achievable distance between the flexed position and the extended position of a particular muscle group, respectively. The score 1 corresponds most important, whereas the score 3 corresponds least important. 


\section{Tables}

Table 1

\section{Timing and type of physiotherapy}

\begin{tabular}{|c|c|c|c|c|}
\hline & $\begin{array}{l}\text { SUPER ACUTE PHASE (0- } \\
24 \text { HOURS) }\end{array}$ & $\begin{array}{l}\text { ACUTE PHASE (24-72 } \\
\text { HOURS) }\end{array}$ & $\begin{array}{l}\text { SUB ACUTE PHASE (4-21 } \\
\text { DAYS) }\end{array}$ & $\begin{array}{l}\text { POST ACUTE PHASE (>21 } \\
\text { DAYS) } \\
\end{array}$ \\
\hline RICE & $10 / 22-45.5 \%$ & $9 / 22-40.9 \%$ & $2 / 22-9.0 \%$ & $1 / 22-4.5 \%$ \\
\hline Megapulse Ultrasound & $1 / 22-4.5 \%$ & $2 / 22-9.0 \%$ & 0 & 0 \\
\hline Passive movement & $2 / 22-9.0 \%$ & $2 / 22-9.0 \%$ & 0 & 0 \\
\hline Walking aids & $2 / 22-9.0 \%$ & $3 / 22-13.6 \%$ & $2 / 22-9.0 \%$ & $4 / 22-18.2 \%$ \\
\hline Mobilisation & 0 & $2 / 22-9.0 \%$ & $4 / 22-18.2 \%$ & $3 / 22-13.6 \%$ \\
\hline Isometric strengthening & 0 & $2 / 22-9.0 \%$ & $2 / 22-9.0 \%$ & 0 \\
\hline Strengthening & 0 & 0 & $2 / 22-9.0 \%$ & $5 / 22-22.7 \%$ \\
\hline Exercise & 0 & $3 / 22-13.6 \%$ & $3 / 22-13.6 \%$ & $5 / 22-22.7 \%$ \\
\hline Ultrasound & 0 & 0 & $9 / 22-40.9 \%$ & $2 / 22-9.0 \%$ \\
\hline PSWD & 0 & $2 / 22-9.0 \%$ & $3 / 22-13.6 \%$ & 0 \\
\hline Hydrotherapy & 0 & 0 & $3 / 22-13.6 \%$ & $1 / 22-4.5 \%$ \\
\hline Active muscle contraction & 0 & 0 & $1 / 22-4.5 \%$ & 0 \\
\hline ROM exercise & 0 & $1 / 22-4.5 \%$ & $3 / 22-13.6 \%$ & $2 / 22-9.0 \%$ \\
\hline Massage & 0 & 0 & $1 / 22-4.5 \%$ & 0 \\
\hline Functional training & 0 & 0 & 0 & $2 / 22-9.0 \%$ \\
\hline Stretching & 0 & 0 & $6 / 22-27.3 \%$ & $4 / 22-18.2 \%$ \\
\hline Re-assessment & 0 & 0 & 0 & $3 / 22-13.6 \%$ \\
\hline No intervention & $10 / 22-45.5 \%$ & $7 / 22-31.8 \%$ & $4 / 22-18.2 \%$ & $7 / 22-31.8 \%$ \\
\hline
\end{tabular}




\section{Legends}

Table 1

\section{Timing and type of physiotherapy}

Lists the recommended type of physiotherapy at various time intervals: 0-24hrs, 24-72hrs, 4-21 days, and >21 days. Data show number of responders as well as the frequency in percentages. 